\title{
Emergence of Dirac and quantum spin Hall states in fluorinated monolayer As and AsSb
}

\author{
Qingyun Zhang and Udo Schwingenschlögl* \\ King Abdullah University of Science and Technology (KAUST), Physical Science and Engineering Division (PSE), \\ Thuwal 23955-6900, Saudi Arabia \\ (Received 6 November 2015; published 21 January 2016)
}

\begin{abstract}
Using first-principles calculations, we investigate the electronic and vibrational properties of monolayer As and AsSb. While the pristine monolayers are semiconductors (direct band gap at the $\Gamma$ point), fluorination results in Dirac cones at the K points. Fluorinated monolayer As shows a band gap of $0.16 \mathrm{eV}$ due to spin-orbit coupling, and fluorinated monolayer AsSb a larger band gap of $0.37 \mathrm{eV}$ due to inversion symmetry breaking. Spin-orbit coupling induces spin splitting similar to monolayer $\mathrm{MoS}_{2}$. Phonon calculations confirm that both materials are dynamically stable. Calculations of the edge states of nanoribbons by the tight-binding method demonstrate that fluorinated monolayer As is topologically nontrivial in contrast to fluorinated monolayer AsSb.
\end{abstract}

DOI: 10.1103/PhysRevB.93.045312

\section{INTRODUCTION}

Two-dimensional materials have a multitude of exciting applications, such as ultimately thin transistors, and pave the way to new device concepts [1]. Since the discovery of graphene in 2004, progress in chemical vapor deposition and exfoliation techniques has led to various new materials, such as monolayer (ML) hexagonal boron nitride [2], $\mathrm{MoS}_{2}[3,4]$, $\mathrm{WS}_{2}$ [5], and silicene [6,7]. Inspired by the special properties of graphene, both silicene and germanene have been under intense study for several years. Except for a buckling, the structure and electronic states of these two materials are similar to graphene, although the band gap is larger and tunable [8,9]. However, the fabrication remains a major challenge despite many efforts [6,10-12]. Recently, ML Sn, the next element in this group of the periodic table, and functionalized ML Sn have been predicted to be large-gap quantum spin Hall [13] and quantum anomalous Hall insulators [14], respectively.

Attention to the neighboring group of the periodic table has been triggered mainly by black phosphorus, which can be used to fabricate thin-film field effect transistors with an excellent room temperature on/off ratio, current saturation (higher than in graphene), and field effect mobility (higher than in ML $\mathrm{MoS}_{2}$ ) [15,16]. Earlier, Bi thin films have shown a fascinating interplay of strong spin-orbit coupling and quantum confinement [17], and the surface states have possible applications in spintronics [18,19]. Being topologically nontrivial, $\mathrm{Bi}$ [20-22] and $\mathrm{Sb}$ [23-25] thin films are of interest ever since the rise of topological insulators, whereas As and AsSb have begun capturing attention only very recently [26-29]. Having hexagonal layered structures similar to bulk $\mathrm{Bi}$ and $\mathrm{Sb}$, it is believed that ML As and AsSb can be fabricated by mechanical and/or chemical methods due to a weak interlayer interaction. The spin-orbit coupling in the two compounds is stronger than in graphene or silicene, which might lead to a nontrivial ground state. Using first-principles calculations, we therefore investigate the electronic and vibrational properties of pristine and fluorinated ML As and AsSb. Phonon calculations are used to study the stability of the materials, and the edge states of nanoribbons are addressed by the tight-binding technique.

*Udo.Schwingenschlogl@kaust.edu.sa

\section{COMPUTATIONAL DETAILS}

For the structure relaxation and subsequent band structure calculations we use the Vienna Ab Initio Simulation Package (VASP) [30]. The generalized gradient approximation [PerdewBurke-Ernzerhof (PBE) flavor] is employed for the exchange correlation functional, the kinetic energy cutoff is set to $500 \mathrm{eV}$, and a $16 \times 16 \times 1 k$ mesh is adopted. Spin-orbit coupling is not included during the structure relaxation but is afterwards added in the band structure calculations. Vacuum layers of $18 \AA$ thickness are adopted to avoid interaction between the top and bottom surfaces in the slab models. The structure relaxation is stopped when the forces on all atoms have declined to less than $0.01 \mathrm{eV} / \AA$ A. Phonon spectra are calculated for a $4 \times 4 \times 1$ supercell by density functional perturbation theory using VASP and PHONOPY [31].

\section{RESULTS AND DISCUSSION}

A side view of pristine ML As is shown in Fig. 1(a). Every atom has three nearest neighbors, forming a pyramidal buckled structure. Figures 1(b) and 1(c) illustrate the structure after fluorination. The hexagonal two-dimensional Brillouin zone is shown in Fig. 1(d). For pristine ML As we obtain for the lattice constant, bond angle, and buckling values $3.61 \AA$, $91.97^{\circ}$, and $1.40 \AA$, respectively, while for pristine ML AsSb the corresponding values are $3.87 \AA, 91.47^{\circ}$, and $1.52 \AA$. The lattice constants of bulk As and AsSb amount to $3.75 \AA$ [32] and $4.03 \AA$ [33], respectively, which implies that the buckling is enhanced from bulk to ML due to the absence of interlayer interaction. Fluorination flattens the structures of ML As and AsSb with lattice constants of $4.57 \AA$ and $4.86 \AA$, respectively.

Band structures and densities of states are shown in Figs. 2(a)-2(d). Pristine ML As is an indirect band-gap semiconductor with the valence band maximum and conduction band minimum at the $\Gamma$ point and along the $\Gamma-\mathrm{M}$ highsymmetry line, respectively. In the vicinity of the Fermi level the bands are mostly due to $p$ states, with small admixtures of $s$ states. Since bulk As is a semimetal, thin films will experience a transition of the band structure as a function of the number of atomic layers. The results for pristine ML AsSb are similar except for a splitting of the bands at low-symmetry $k$ points (absence of inversion symmetry). Indirect band gaps of 1.47 and $1.27 \mathrm{eV}$ are found for pristine ML As and AsSb, 
(a)

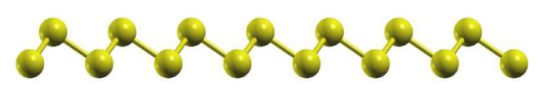

(c)

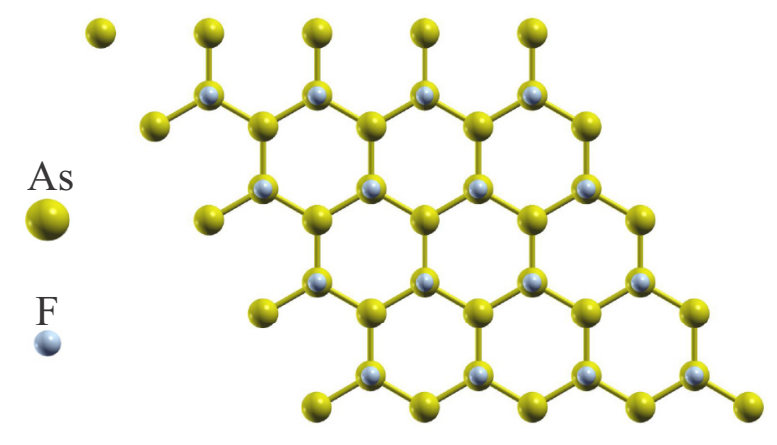

(b)

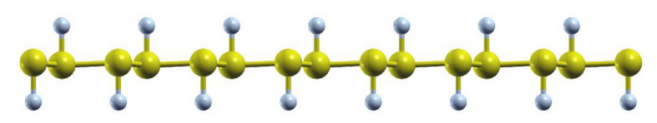

(d) attice.

FIG. 1. (a) Atomic structure of pristine ML As. (b) Side view and (c) top view of fluorinated ML As. (d) Brillouin zone of the hexagonal

respectively, which agrees well with recent theoretical studies [26-29]. More accurate (enhanced) values of the band gap are obtained by the HSE06 hybrid functional [see the red lines in Figs. 2(a), 2(c), 2(e), and 2(g)], amounting to 2.06 and $1.78 \mathrm{eV}$. We note that fluorinated ML As [Figs. 2(e) and 2(f)] shows Dirac cones at the $\mathrm{K}$ points with a band gap of $0.16 \mathrm{eV}$ when the spin-orbit coupling is turned on (much larger than in silicene [8,9]). For fluorinated ML AsSb [Figs. 2(g) and 2(h)] also a gapped Dirac cone is found, however, even without spin-orbit coupling, as a consequence of the inversion symmetry breaking. The spin-orbit coupling results in an out-of-plane spin splitting in the vicinity of the $\mathrm{K}$ points, similar to $\mathrm{ML} \mathrm{MoS}_{2}$. Comparison of the HSE06 and PBE calculations shows for fluorinated ML As and AsSb only slight changes in the size of the band gap, while the slope is enhanced for the hybrid functional. The densities of states of the fluorinated compounds demonstrate that the Dirac states are almost purely due to the As and $\mathrm{Sb}$ atoms. Figure 3(a) shows that applied strain tunes the size of the band gap. For fluorinated ML As we find a moderate enhancement, while for fluorinated ML AsSb the band gap decreases quickly under tensile strain. According to Fig. 3(b), the spin splitting at the $\mathrm{K}$ valleys in fluorinated ML AsSb is larger for the conduction band edge than for the valence band edge, in strict contrast to $\mathrm{ML} \mathrm{MoS}_{2}$, and grows slightly under tensile strain.

The phonon spectra in Fig. 4 show only minor negative frequencies for the fluorinated compounds, which are wellknown artifacts of the numerics. Therefore, all four materials under investigation are dynamically stable. For pristine ML As [Fig. 4(a)] two acoustic phonon modes correspond to inplane vibrations and one (with lower energy) to out-of-plane vibrations. We note that two optical modes are degenerate at the $\Gamma$ point, which correspond to in-plane antiphase displacements of the As atoms. Fluorinated ML As [Fig. 4(c)] shows two high-frequency optical phonon modes, which are due to inplane antiphase displacements of the $\mathrm{F}$ and As atoms with respect to each other. The behavior of ML AsSb is similar with slightly softened modes [Figs. 4(b) and 4(d)]. We also calculate the formation energy

$$
E_{f}=\left(E_{\mathrm{total}}-n_{\mathrm{As}} E_{\mathrm{As}}-n_{\mathrm{Sb}} E_{\mathrm{Sb}}-n_{\mathrm{F}} E_{\mathrm{F}}\right) /\left(n_{\mathrm{As}}+n_{\mathrm{Sb}}+n_{\mathrm{F}}\right) \text {, }
$$

where $E_{\text {total }}$ is the total energy of the compound, $n_{\mathrm{As}}, n_{\mathrm{Sb}}$, and $n_{\mathrm{F}}$ are the numbers of $\mathrm{As}, \mathrm{Sb}$, and $\mathrm{F}$ atoms, and $E_{\mathrm{As}}, E_{\mathrm{Sb}}$, and $E_{\mathrm{F}}$ are the chemical potentials obtained from ML As, ML Sb, and the $\mathrm{F}_{2}$ molecule, respectively. We find formation energies of -1.19 and $-1.26 \mathrm{eV}$ for fluorinated ML As and AsSb, respectively.

We next investigate the bonding character of (fluorinated) ML As in Fig. 5 by charge density difference plots with respect to isolated As atoms. Figure 5(a) shows a $s p^{3}$ hybridization reflecting the buckled structure. After fluorination we obtain $s p^{2}$ and $p_{z}$ orbitals instead (flat structure), which form $\sigma$ and $\pi$ bonds analogous to graphene. Thus, an effective Hamiltonian with spin-orbit coupling can be used to describe the material:

$$
\begin{aligned}
H_{\tau}^{\mathrm{eff}}= & v_{F}\left(\tau p_{x} \hat{\sigma}_{x}+p_{y} \hat{\sigma}_{y}\right)+\frac{\Delta}{2} \hat{\sigma}_{z} \\
& +\frac{1}{2} \tau\left(\frac{\lambda_{1}+\lambda_{2}}{2} \hat{\sigma}_{z}+\frac{\lambda_{1}-\lambda_{2}}{2}\right) \hat{s}_{z},
\end{aligned}
$$

where $v_{F}, \Delta, \lambda_{1 / 2}, \tau, \hat{\sigma}_{x / y / z}$, and $\hat{s}_{z}$ denote the Fermi velocity, energy gap due to inversion symmetry breaking, strength of the spin-orbit coupling, valley index, Pauli matrices for the two basis functions, and spin, respectively. The intrinsic Rashba spin-orbit coupling is omitted, since the fluorinated compounds have planar structures. By fitting $H_{\tau}^{\text {eff }}$ to our first-principles band structures we obtain $v_{F}=7.16 \times 10^{5}$ $\left(6.53 \times 10^{5}\right) \mathrm{m} / \mathrm{s}, \Delta=0(0.37) \mathrm{eV}, \lambda_{1}=0.16(0.33) \mathrm{eV}$, and $\lambda_{2}=0.16(0.19) \mathrm{eV}$ for fluorinated ML As (AsSb).

Since in fluorinated ML As the band gap is opened exclusively by the spin-orbit coupling, it is topologically nontrivial similar to silicene. On the other hand, for 


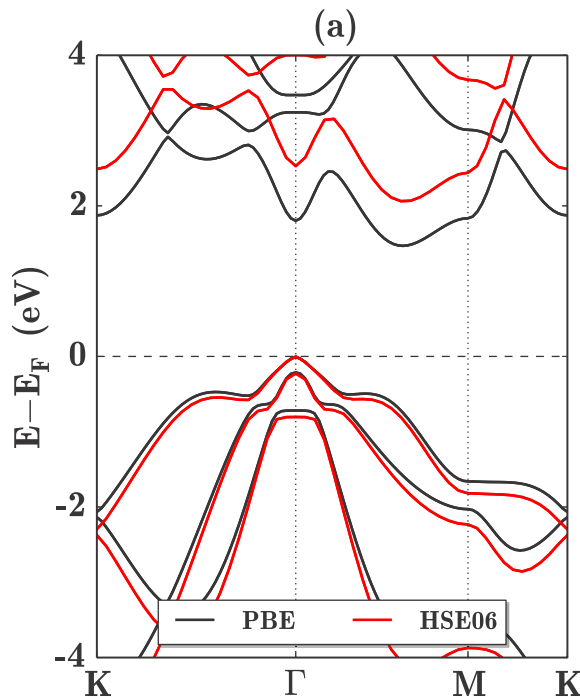

(b)

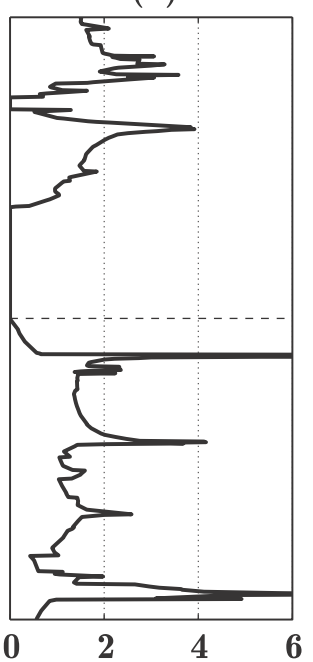

(e)

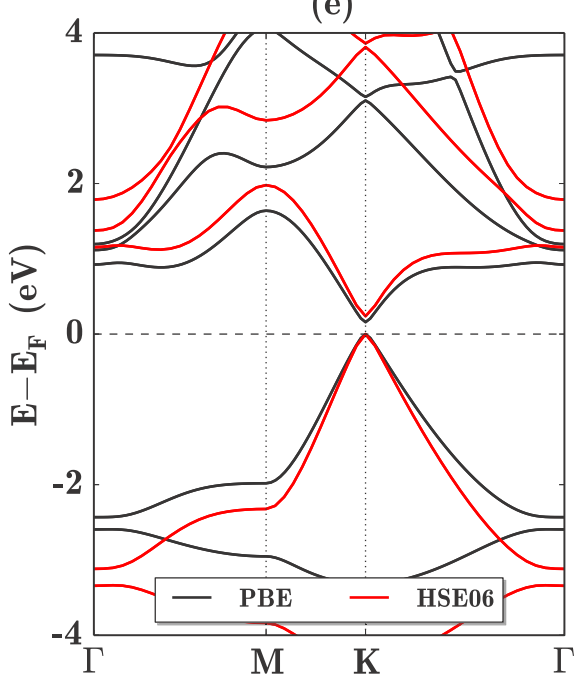

(f)

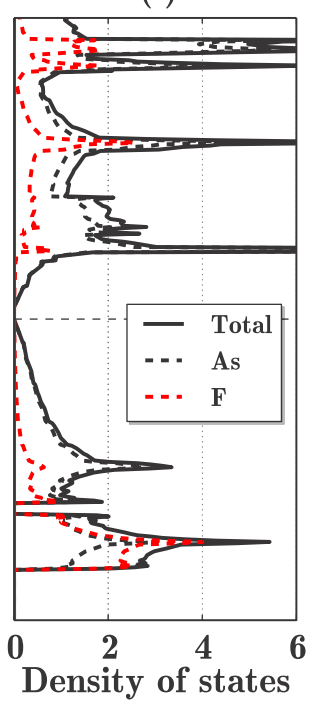

$\left(\mathrm{eV}^{-1}\right)$ (c)

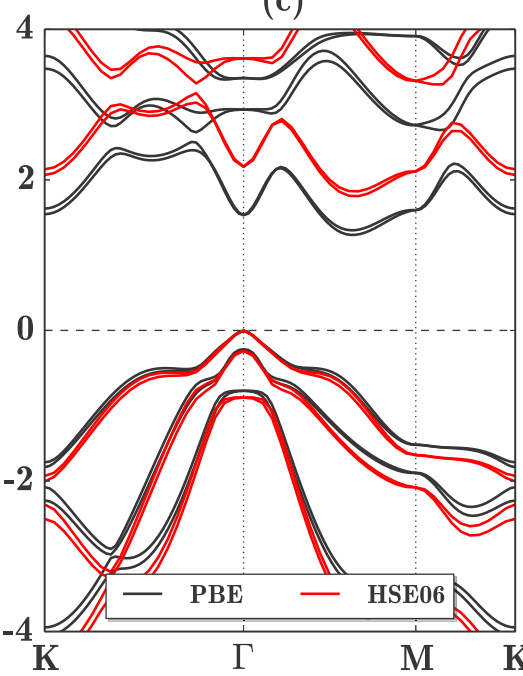

(g)

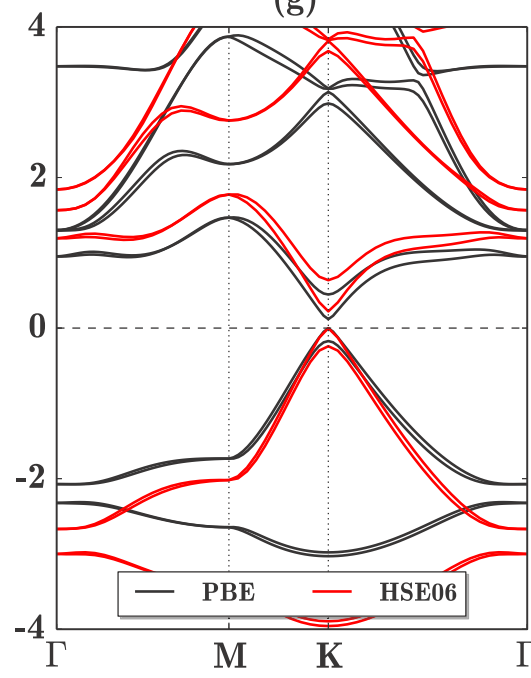

(d)

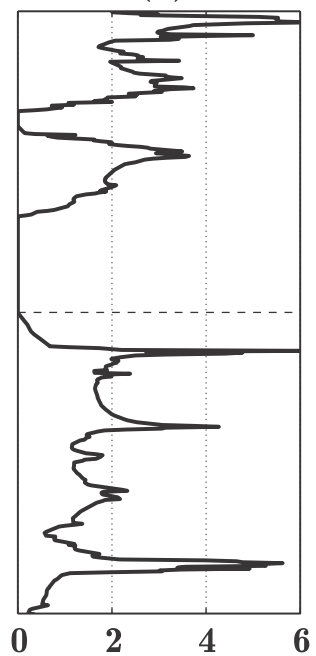

(h)

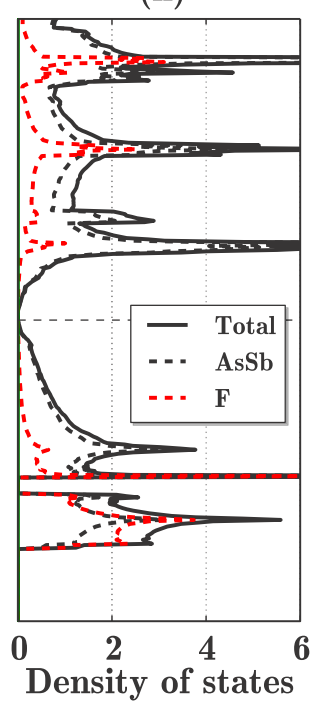

$\left(\mathrm{eV}^{-1}\right)$

FIG. 2. Band structure and density of states of $(\mathrm{a}, \mathrm{b})$ pristine ML As, (c,d) pristine ML AsSb, (e,f) fluorinated ML As, and (g,h) fluorinated ML AsSb.

(a)

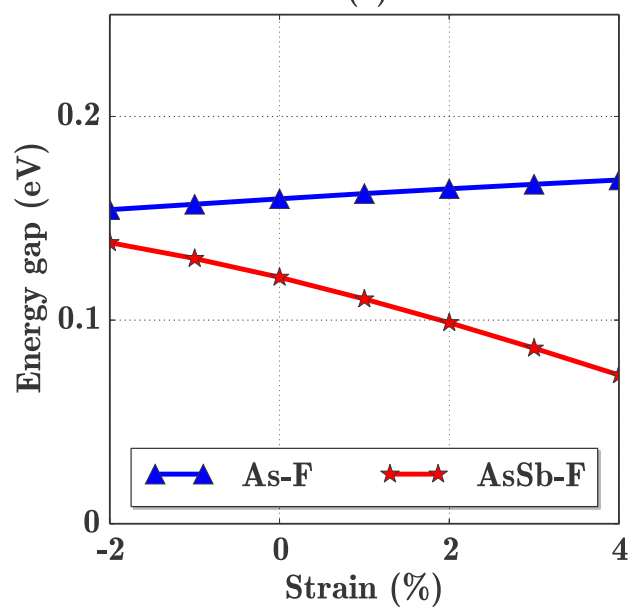

(b)

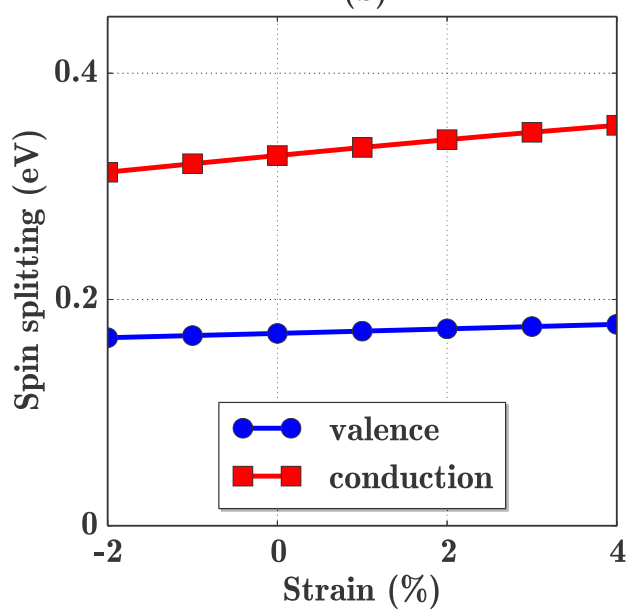

FIG. 3. Effect of strain: (a) Size of the band gap in fluorinated ML As and AsSb, and (b) spin splitting at the K valleys in fluorinated ML AsSb. 
(a)

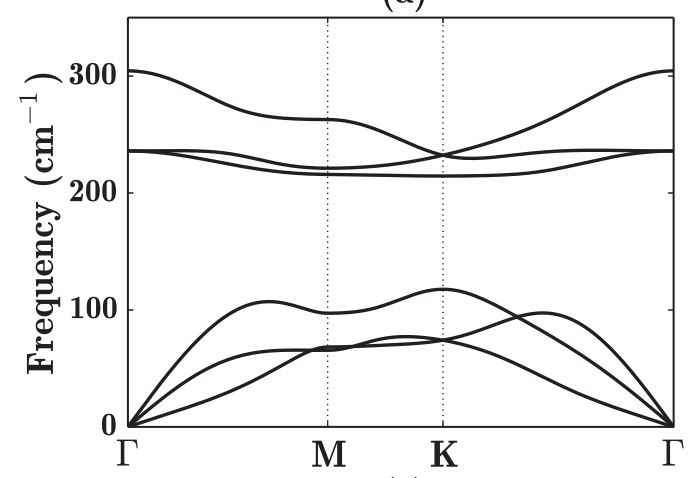

(c)

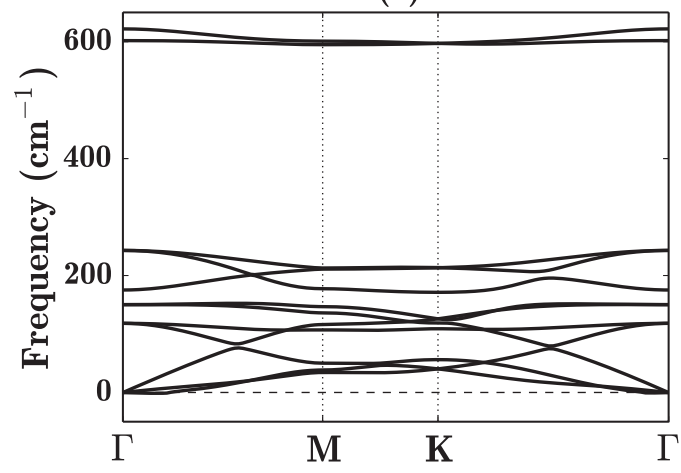

(b)

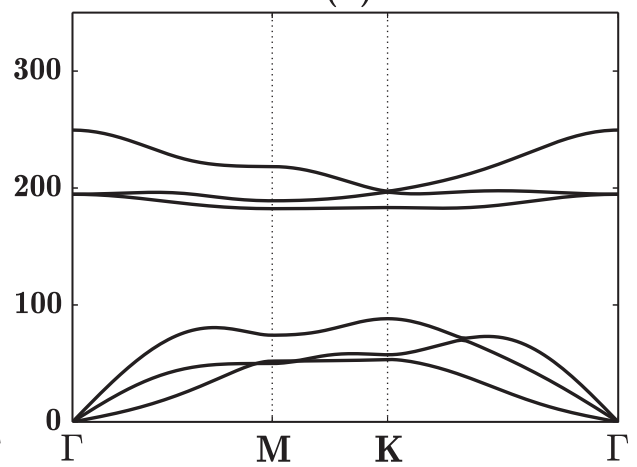

(d)

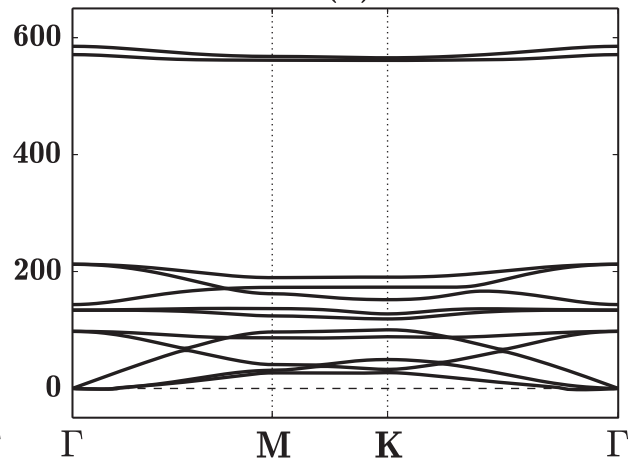

FIG. 4. Phonon spectrum of (a) pristine ML As, (b) pristine ML AsSb, (c) fluorinated ML As, and (d) fluorinated ML AsSb.

fluorinated ML AsSb the band gap is already opened by the inequality of the two sublattices. The spin-orbit coupling just induces an additional spin splitting at the conduction and valence band edges, which is also found in other inversion symmetry broken systems with hexagonal lattices, such as ML $\mathrm{MoS}_{2}$. We notice that this splitting is not large enough to close the band gap, which implies that the system is a trivial semiconductor. Since the spin-up and -down bands are decoupled, the $Z_{2}$ index is identical to the spin Chern number $C_{s}$ modulo 2 [34]. From $H_{\tau}^{\text {eff }}$, the spin Chern number can be calculated to be $\left[1-\operatorname{sgn}\left(\Delta-\frac{\lambda_{1}+\lambda_{2}}{2}\right)\right] / 2$, which is 1 and 0 for fluorinated ML As and AsSb, respectively. To further confirm the topological properties, we calculate the band structures of zigzag nanoribbons by means of the tight-binding method based on maximally localized Wannier functions, generated by the WANNIER90 code [35]. For fluorinated ML
As [Fig. 6(a)], nontrivial edge states are found inside the band gap with spin-up and -down channels propagating in opposite directions, whereas for fluorinated ML AsSb [Fig. 6(b)], the edge states inside the band gap are trivial, since there are two crossings with the Fermi level for each spin band.

In order to address possible disorder effects in ML AsSb, we employ the special quasirandom structure approach [36,37]. Neglecting spin-orbit coupling in the calculations, we obtain band gaps of $0.021,0.016$, and $0.011 \mathrm{eV}$ for system sizes (lattice constants) of $19.3,24.1$, and $33.8 \AA$, respectively, which shows that the band gap converges to zero for increasing disorder. We can conclude that disorder weakens the effects of the inversion symmetry breaking characteristic for the ordered structure. As a consequence, growing disorder brings fluorinated ML AsSb gradually back to a topologically nontrivial state. (a)

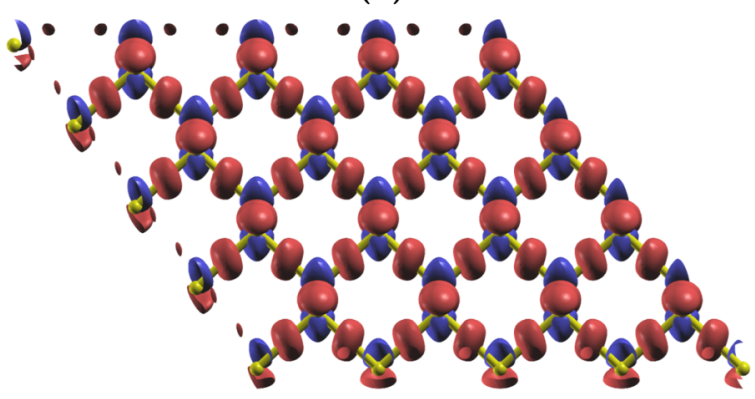

(b)

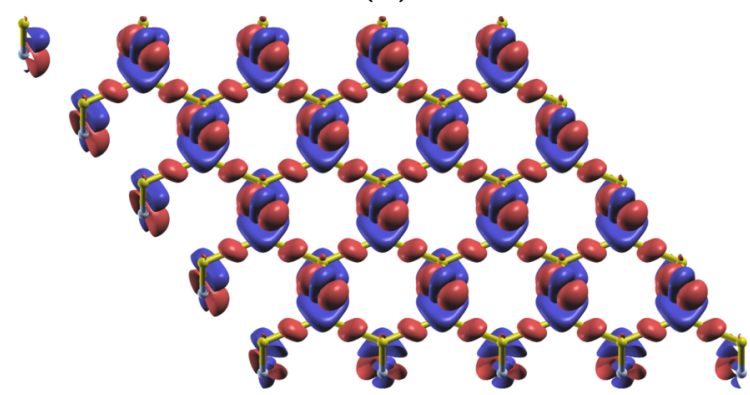

FIG. 5. Charge density difference of (a) pristine ML As and (b) fluorinated ML As with respect to isolated As atoms (isovalue: 0.05 electrons $/ \AA^{3}$ ). 
(a)

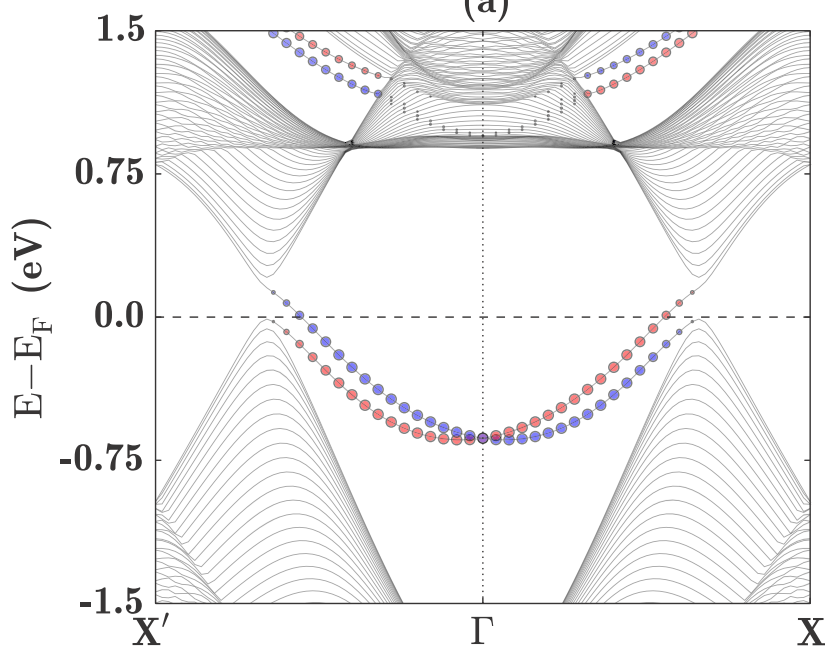

(b)

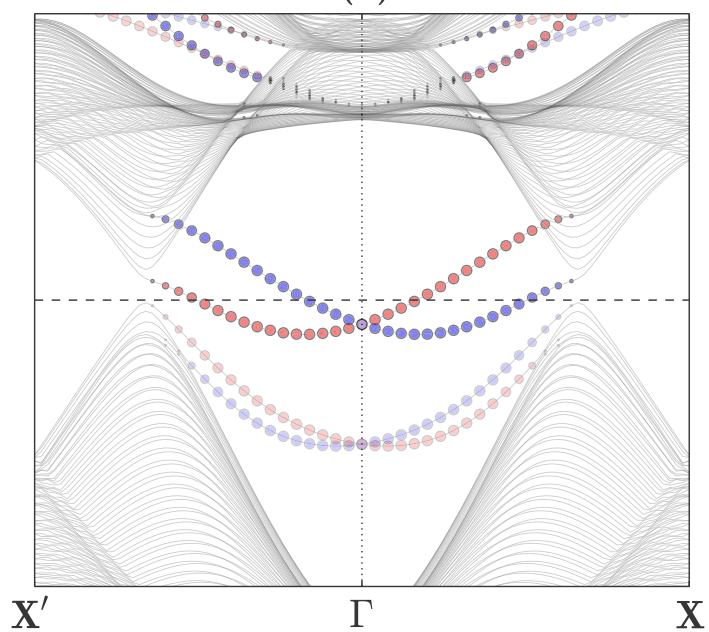

FIG. 6. Tight-binding band structure of fluorinated ML (a) As and (b) AsSb zigzag nanoribbons. The size of the circles quantifies the projection on the edge atoms and red/blue color corresponds to spin-up/down.

\section{CONCLUSION}

In conclusion, we have demonstrated that pristine ML As and $\mathrm{AsSb}$ are indirect band-gap semiconductors (1.47 and $1.27 \mathrm{eV}$, respectively). On the other hand, the fluorinated materials are direct band-gap semiconductors with Dirac cones at the $\mathrm{K}$ points. For fluorinated ML As the origin of the band gap is the spin-orbit coupling, while for fluorinated ML $\mathrm{AsSb}$ it is the inversion symmetry breaking. (The spin-orbit coupling induces only an additional spin splitting.) As a consequence, fluorinated ML As is topologically nontrivial in contrast to fluorinated ML AsSb, which is confirmed by the edge states obtained for nanoribbons. Phonon calculations indicate stability of all the materials under investigation. We have also studied functionalization with $\mathrm{H}$ and other halide atoms and find that the band structures are similar to those of the fluorinated compounds, but the structural stability is reduced. Fluorinated ML As appears to be an ideal replacement for silicene, because it does not suffer from the structural instability of this material while sharing all the relevant features of the electronic states.

\section{ACKNOWLEDGMENTS}

We thank Dr. Z. M. Shi for helpful discussions. The research reported in this publication was supported by funding from King Abdullah University of Science and Technology (KAUST).
[1] G. Fiori, F. Bonaccorso, G. Iannaccone, T. Palacios, D. Neumaier, A. Seabaugh, S. K. Banerjee, and L. Colombo, Nat. Nanotechnol. 9, 768 (2014).

[2] D. Golberg, Y. Bando, Y. Huang, T. Terao, M. Mitome, C. Tang, and C. Zhi, ACS Nano 4, 2979 (2010).

[3] K. F. Mak, C. Lee, J. Hone, J. Shan, and T. F. Heinz, Phys. Rev. Lett. 105, 136805 (2010).

[4] B. Radisavljevic, A. Radenovic, J. Brivio, V. Giacometti, and A. Kis, Nat. Nanotechnol. 6, 147 (2011).

[5] Q. H. Wang, K. Kalantar-Zadeh, A. Kis, J. N. Coleman, and M. S. Strano, Nat. Nanotechnol. 7, 699 (2012).

[6] A. Kara, H. Enriquez, A. P. Seitsonen, L. C. L. Y. Voon, S. Vizzini, B. Aufray, and H. Oughaddou, Surf. Sci. Rep. 67, 1 (2012).

[7] L. Tao, E. Cinquanta, D. Chiappe, C. Grazianetti, M. Fanciulli, M. Dubey, A. Molle, and D. Akinwande, Nat. Nanotechnol. 10, 227 (2015).

[8] S. Cahangirov, M. Topsakal, E. Aktürk, H. Şahin, and S. Ciraci, Phys. Rev. Lett. 102, 236804 (2009).

[9] C.-C. Liu, W. X. Feng, and Y. G. Yao, Phys. Rev. Lett. 107, 076802 (2011).
[10] P. Vogt, P. De Padova, C. Quaresima, J. Avila, E. Frantzeskakis, M. C. Asensio, A. Resta, B. Ealet, and G. Le Lay, Phys. Rev. Lett. 108, 155501 (2012).

[11] B. Feng, Z. Ding, S. Meng, Y. Yao, X. He, P. Cheng, L. Chen, and K. Wu, Nano Lett. 12, 3507 (2012).

[12] C.-L. Lin, R. Arafune, K. Kawahara, N. Tsukahara, E. Minamitani, Y. Kim, N. Takagi, and M. Kawai, Appl. Phys. Express 5, 045802 (2012).

[13] Y. Xu, B. H. Yan, H.-J. Zhang, J. Wang, G. Xu, P. Z. Tang, W. H. Duan, and S.-C. Zhang, Phys. Rev. Lett. 111, 136804 (2013).

[14] S.-C. Wu, G. C. Shan, and B. H. Yan, Phys. Rev. Lett. 113, 256401 (2014).

[15] H. Liu, A. T. Neal, Z. Zhu, Z. Luo, X. Xu, D. Tománek, and P. D. Ye, ACS Nano 8, 4033 (2014).

[16] L. Li, Y. Yu, G. J. Ye, Q. Ge, X. Ou, H. Wu, D. Feng, X. H. Chen, and Y. Zhang, Nat. Nanotechnol. 9, 372 (2014).

[17] C. A. Hoffman, J. R. Meyer, F. J. Bartoli, A. Di Venere, X. J. Yi, C. L. Hou, H. C. Wang, J. B. Ketterson, and G. K. Wong, Phys. Rev. B 48, 11431 (1993). 
[18] T. Hirahara, T. Nagao, I. Matsuda, G. Bihlmayer, E. V. Chulkov, Y. M. Koroteev, P. M. Echenique, M. Saito, and S. Hasegawa, Phys. Rev. Lett. 97, 146803 (2006).

[19] Y. M. Koroteev, G. Bihlmayer, E. V. Chulkov, and S. Blügel, Phys. Rev. B 77, 045428 (2008).

[20] D. Hsieh, D. Qian, L. Wray, Y. Xia, Y. S. Hor, R. J. Cava, and M. Z. Hasan, Nature (London) 452, 970 (2008).

[21] M. Wada, S. Murakami, F. Freimuth, and G. Bihlmayer, Phys. Rev. B 83, 121310(R) (2011).

[22] Z. Liu, C.-X. Liu, Y.-S. Wu, W.-H. Duan, F. Liu, and J. Wu, Phys. Rev. Lett. 107, 136805 (2011).

[23] G. Bian, T. Miller, and T.-C. Chiang, Phys. Rev. Lett. 107, 036802 (2011).

[24] P. F. Zhang, Z. Liu, W. Duan, F. Liu, and J. Wu, Phys. Rev. B 85, 201410(R) (2012).

[25] G. Yao, Z. Luo, F. Pan, W. Xu, Y. P. Feng, and X. Wang, Sci. Rep. 3, 2010 (2013).

[26] C. Kamal and M. Ezawa, Phys. Rev. B 91, 085423 (2015).
[27] Z. Zhu, J. Guan, and D. Tománek, Phys. Rev. B 91, 161404(R) (2015).

[28] S. L. Zhang, Z. Yan, Y. F. Li, Z. F. Chen, and H. B. Zeng, Angew. Chem. Int. Ed. 54, 3112 (2015).

[29] L. Z. Kou, Y. D. Ma, X. Tan, T. Frauenheim, A. J. Du, and S. Smith, J. Phys. Chem. C 119, 6918 (2015).

[30] G. Kresse and J. Hafner, Phys. Rev. B 47, 558 (1993).

[31] A. Togo and I. Tanaka, Scr. Mater. 108, 1 (2015).

[32] D. Schiferl and C. S. Barrett, J. Appl. Crystallogr. 2, 30 (1969).

[33] P. Bayliss, Am. Mineral. 76, 257 (1991).

[34] M. Ezawa, Eur. Phys. J. B 85, 363 (2012).

[35] A. A. Mostofi, J. R. Yates, Y.-S. Lee, I. Souza, D. Vanderbilt, and N. Marzari, Comput. Phys. Commun. 178, 685 (2008).

[36] A. Zunger, S.-H. Wei, L. G. Ferreira, and J. E. Bernard, Phys. Rev. Lett. 65, 353 (1990).

[37] A. van de Walle, P. Tiwary, M. de Jong, D. L. Olmsted, M. Asta, A. Dick, D. Shin, Y. Wang, L.-Q. Chen, and Z.-K. Liu, CALPHAD 42, 13 (2013). 